\title{
Solvability of integrodifferential problems via fixed point theory in $b$-metric spaces
}

\author{
Monica Cosentino ${ }^{1}$, Mohamed Jleli $^{2}$, Bessem Samet ${ }^{2 *}$ and Calogero Vetro
}

${ }^{*}$ Correspondence:

bsamet@ksu.edu.sa

2Department of Mathematics,

College of Science, King Saud

University, P.O. Box 2455, Riyadh,

11451, Saudi Arabia

Full list of author information is

available at the end of the article

\begin{abstract}
The purpose of this paper is to study the existence of solutions set of integrodifferential problems in Banach spaces. We obtain our results by using fixed point theorems for multivalued mappings, under new contractive conditions, in the setting of complete $b$-metric spaces. Also, we present a data dependence theorem for the solutions set of fixed point problems.
\end{abstract}

MSC: $47 \mathrm{H} 10 ; 34 \mathrm{~A} 60$

Keywords: $b$-metric space; differential inclusion; fixed point; multivalued mapping

\section{Introduction}

Measure theory is a classical topic in mathematical analysis which is usually studied in the setting of real and complex numbers and functions. Indeed, measures have applications in the foundations of integration, probability and ergodic theories. On the other hand, theory of multivalued mappings has an important role in various branches of mathematics because of its applications in optimal control problems involving integrodifferential inclusions. As a matter of fact, the theory of integrodifferential equations and inclusions has undergone rapid development over the last decades (see, for instance, [1] and the references therein). Indeed, this theory has increased its significance in modern applied mathematical models of real processes arising in many engineering and scientific disciplines such as physics, biology, economics, signal processing and data fitting.

Notice that in the literature there are many papers focusing on the solution of differential problems approached via fixed point theory (see, for example, [2-4] and the references therein). On the other hand, it is well known that metric spaces and their generalizations furnish an useful tool for the study of multivalued mappings. In this regard, Nadler [5] was the first author who combined the ideas of contractions and multivalued mappings by providing a fixed point existence result.

Theorem 1.1 ([5]) Let $(X, d)$ be a complete metric space and let $T: X \rightarrow C B(X)$ be a multivalued mapping satisfying $H(T x, T y) \leq k d(x, y)$ for all $x, y \in X$, where $k$ is a constant such that $k \in(0,1)$, and $C B(X)$ denotes the family of non-empty, closed and bounded subsets of $X$. Then $T$ has a fixed point, that is, there exists a point $u \in X$ such that $u \in T u$.

Later on, many generalizations, extensions and applications of this theorem have appeared in the literature (see, for instance, [6-13]). In this literature review, we start from

(c) 2015 Cosentino et al.; licensee Springer. This article is distributed under the terms of the Creative Commons Attribution 4.0 International License (http://creativecommons.org/licenses/by/4.0/), which permits unrestricted use, distribution, and reproduction in any medium, provided you give appropriate credit to the original author(s) and the source, provide a link to the Creative Commons license, and indicate if changes were made. 
looking at the paper of Wardowski [14] who introduced a new concept of contraction, called $F$-contraction. Consequently, Wardowski proved fixed point theorems generalizing the Banach-Caccioppoli fixed point theorem (of which Theorem 1.1 is the multivalued version) in a new way than in the previous known theorems of the same class. Subsequently, Sgroi and Vetro [15] extended Wardowski's ideas to the case of multivalued mappings and studied the solution of certain functional and integral equations under a suitable set of hypotheses. Yet another inspiration for our work comes from Feng and Liu's paper [16] providing useful tools to establish both global and local fixed point theorems. Finally, we recall that the concept of metric space has been generalized in many directions to include measurements in a much more general sense. Here, we focus our attention on the notion of $b$-metric spaces, which are metric spaces satisfying a relaxed form of triangle inequality, see Czerwik [17] and Bakhtin [18]. Several researchers followed the idea of Czerwik and proved interesting results [19-25].

In this paper, we study the existence of solutions for certain integral problems of Fredholm type in Banach spaces. Also, we present a data dependence theorem for the solutions set of fixed point problems. We obtain our results by using fixed point theorems for multivalued mappings, under new contractive conditions, in the setting of complete $b$-metric spaces. Clearly, the presented theorems extend well-known results in the literature to $b$ metric spaces.

\section{Preliminaries}

In this section, we collect some basic definitions, lemmas and notations which will be used throughout the paper (see $[17,18,26,27]$ and the references therein). Let $\mathbb{R}^{+}$denote the set of all nonnegative real numbers and $\mathbb{N}$ denote the set of positive integers.

Definition 2.1 Let $X$ be a non-empty set and let $s \geq 1$ be a given real number. A function $d: X \times X \rightarrow \mathbb{R}^{+}$is said to be a $b$-metric if and only if for all $x, y, z \in X$ the following conditions are satisfied:

(1) $d(x, y)=0$ if and only if $x=y$;

(2) $d(x, y)=d(y, x)$;

(3) $d(x, z) \leq s[d(x, y)+d(y, z)]$.

Then the triplet $(X, d, s)$ is called a $b$-metric space.

Clearly, a (standard) metric space is also a $b$-metric space, but the converse is not always true.

Example 2.2 Let $X=[0,1]$ and $d: X \times X \rightarrow \mathbb{R}^{+}$be defined by $d(x, y)=|x-y|^{2}$ for all $x, y \in X$. Clearly, $(X, d, 2)$ is a $b$-metric space that is not a metric space.

Again, let $(X, d, s)$ be a $b$-metric space. The following notions are natural deductions from the corresponding metric versions:

(i) A sequence $\left\{x_{n}\right\} \subseteq X$ converges to $x \in X$ if $\lim _{n \rightarrow+\infty} d\left(x_{n}, x\right)=0$.

(ii) A sequence $\left\{x_{n}\right\} \subseteq X$ is said to be a Cauchy sequence if, for every given $\varepsilon>0$, there exists a positive integer $n(\varepsilon)$ such that $d\left(x_{m}, x_{n}\right)<\varepsilon$ for all $m, n \geq n(\varepsilon)$.

(iii) A $b$-metric space $(X, d, s)$ is said to be complete if and only if each Cauchy sequence converges to some $x \in X$.

Next, we give two significant examples of $b$-metric spaces from the literature. 
Example 2.3 ([19]) Let $p \in(0,1)$. Then the set $l^{p}(\mathbb{R}):=\left\{\left\{x_{n}\right\} \subset \mathbb{R}: \sum_{n=1}^{\infty}\left|x_{n}\right|^{p}<\infty\right\}$ endowed with the functional $d: l^{p}(\mathbb{R}) \times l^{p}(\mathbb{R}) \rightarrow \mathbb{R}$ given by

$$
d\left(\left\{x_{n}\right\},\left\{y_{n}\right\}\right)=\left(\sum_{n=1}^{\infty}\left|x_{n}-y_{n}\right|^{p}\right)^{1 / p}
$$

for all $\left\{x_{n}\right\},\left\{y_{n}\right\} \in l^{p}(\mathbb{R})$ is a $b$-metric space with $s=2^{1 / p}$.

Example 2.4 ([19]) Let $p \in(0,1)$. Then the space $L^{p}([0,1])$ of all real functions $f:[0,1] \rightarrow$ $\mathbb{R}$ such that $\int_{0}^{1}|f(t)|^{p} d t<\infty$ endowed with the functional $d: L^{p}([0,1]) \times L^{p}([0,1]) \rightarrow \mathbb{R}$ given by

$$
d(f, g)=\left(\int_{0}^{1}|f(t)-g(t)|^{p} d t\right)^{1 / p}
$$

for all $f, g \in L^{p}([0,1])$ is a $b$-metric space with $s=2^{1 / p}$.

Now, we give a brief background for multivalued mappings defined in a $b$-metric space $(X, d, s)$. For $A, B \in C B(X)$, define the function $H: C B(X) \times C B(X) \rightarrow \mathbb{R}^{+}$by

$$
H(A, B)=\max \{\delta(A, B), \delta(B, A)\}
$$

where

$$
\delta(A, B)=\sup \{d(a, B), a \in A\}, \quad \delta(B, A)=\sup \{d(b, A), b \in B\}
$$

with

$$
d(a, C)=\inf \{d(a, x), x \in C\}
$$

Note that $H$ is called the Hausdorff $b$-metric induced by the $b$-metric $d$.

We recall the following properties from [17, 24, 27].

Lemma 2.5 Let $(X, d, s)$ be a $b$-metric space. For any $A, B, C \in C B(X)$ and any $x, y \in X$, we have the following:

(i) $d(x, B) \leq d(x, b)$ for any $b \in B$;

(ii) $\delta(A, B) \leq H(A, B)$;

(iii) $d(x, B) \leq H(A, B)$ for any $x \in A$;

(iv) $H(A, A)=0$;

(v) $H(A, B)=H(B, A)$;

(vi) $H(A, C) \leq s[H(A, B)+H(B, C)]$;

(vii) $d(x, A) \leq s[d(x, y)+d(y, A)]$.

Lemma 2.6 Let $(X, d, s)$ be a b-metric space and $A, B \in C B(X)$. Then, for each $h>1$ and for each $a \in A$, there exists $b(a) \in B$ such that $d(a, b(a)) \leq h H(A, B)$. 
Lemma 2.7 Let $(X, d, s)$ be a b-metric space. For $A \in C B(X)$ and $x \in X$, we have

$$
d(x, A)=0 \quad \Longleftrightarrow \quad x \in \bar{A}=A,
$$

where $\bar{A}$ denotes the closure of the set $A$.

We conclude this section with two useful lemmas.

Lemma 2.8 Let $(X, d, s)$ be a b-metric space and let $\left\{x_{n}\right\}$ be a sequence in $X$. If $\lim _{n \rightarrow+\infty} x_{n}=$ $y$ and $\lim _{n \rightarrow+\infty} x_{n}=z$, then $y=z$.

Lemma 2.9 Let $(X, d, s)$ be a $b$-metric space and let $\left\{x_{n}\right\}$ be a sequence in $X$ such that

$$
d\left(x_{n}, x_{n+1}\right) \leq \lambda d\left(x_{n-1}, x_{n}\right)
$$

for some $\lambda \in\left(0, s^{-1}\right)$ and each $n \in \mathbb{N}$. Then $\left\{x_{n}\right\}$ is a Cauchy sequence in $X$.

\section{Fixed point theory in $b$-metric spaces}

\subsection{Wardowski type theorem}

We study the existence of fixed points for multivalued mappings by adapting the ideas in [14] to the $b$-metric setting. The motivation of this research is to solve certain classes of integrodifferential problems. First, inspired by Wardowski [14], we give the following definitions.

Definition 3.1 Let $s \geq 1$ be a real number. We denote by $\mathcal{F}_{s}$ the family of all functions $F: \mathbb{R}^{+} \rightarrow \mathbb{R}$ with the following properties:

(F1) $F$ is strictly increasing;

(F2) for each sequence $\left\{\alpha_{n}\right\} \subset \mathbb{R}^{+}$of positive numbers $\lim _{n \rightarrow+\infty} \alpha_{n}=0$ if and only if $\lim _{n \rightarrow+\infty} F\left(\alpha_{n}\right)=-\infty$

(F3) for each sequence $\left\{\alpha_{n}\right\} \subset \mathbb{R}^{+}$of positive numbers with $\lim _{n \rightarrow+\infty} \alpha_{n}=0$, there exists $k \in(0,1)$ such that $\lim _{n \rightarrow+\infty}\left(\alpha_{n}\right)^{k} F\left(\alpha_{n}\right)=0$;

(F4) for each sequence $\left\{\alpha_{n}\right\} \subset \mathbb{R}^{+}$of positive numbers such that $\tau+F\left(s \alpha_{n}\right) \leq F\left(\alpha_{n-1}\right)$ for all $n \in \mathbb{N}$ and some $\tau \in \mathbb{R}^{+}$, then $\tau+F\left(s^{n} \alpha_{n}\right) \leq F\left(s^{n-1} \alpha_{n-1}\right)$ for all $n \in \mathbb{N}$.

Example 3.2 Let $F: \mathbb{R}^{+} \rightarrow \mathbb{R}$ be defined by $F(x)=x+\ln x$. Clearly, $F$ satisfies (F1)-(F4). Here we show only (F4).

Assume that, for all $n \in \mathbb{N}$ and some $\tau \in \mathbb{R}^{+}$, we have $\tau+s \alpha_{n}+\ln \left(s \alpha_{n}\right) \leq \alpha_{n-1}+\ln \alpha_{n-1}$. Since $x+\ln x$ is an increasing function, then $s \alpha_{n}<\alpha_{n-1}$. Thus

$$
\left(s^{n-1}-1\right) s \alpha_{n}+\ln s^{n-1} \leq\left(s^{n-1}-1\right) \alpha_{n-1}+\ln s^{n-1}
$$

implies that

$$
\begin{aligned}
\tau+s^{n} \alpha_{n}+\ln s^{n} \alpha_{n} & =\tau+s \alpha_{n}+\left(s^{n-1}-1\right) s \alpha_{n}+\ln s^{n-1}+\ln s \alpha_{n} \\
& \leq \alpha_{n-1}+\left(s^{n-1}-1\right) \alpha_{n-1}+\ln s^{n-1}+\ln \alpha_{n-1} \\
& =s^{n-1} \alpha_{n-1}+\ln \left(s^{n-1} \alpha_{n-1}\right)
\end{aligned}
$$


and hence (F4) holds true. Note that also $F: \mathbb{R}^{+} \rightarrow \mathbb{R}$ defined by $F(x)=\ln x$ satisfies (F1)(F4).

Definition 3.3 Let $(X, d, s)$ be a $b$-metric space. A multivalued mapping $T: X \rightarrow C B(X)$ is called an $F$-contraction of Nadler type if there exist $F \in \mathcal{F}_{s}$ and $\tau \in \mathbb{R}^{+}$such that

$$
2 \tau+F(s H(T x, T y)) \leq F(d(x, y))
$$

for all $x, y \in X$ with $T x \neq T y$.

Now, we are ready to state and prove our first main theorem.

Theorem 3.4 Let $(X, d, s)$ be a complete $b$-metric space and let $T: X \rightarrow C B(X)$. Assume that there exists a continuous from the right function $F \in \mathcal{F}_{s}$ and $\tau \in \mathbb{R}^{+}$such that

$$
2 \tau+F(s H(T x, T y)) \leq F(d(x, y))
$$

for all $x, y \in X, T x \neq T y$. Then $T$ has a fixed point.

Proof Let $x_{0} \in X$ be an arbitrary point of $X$ and choose $x_{1} \in T x_{0}$. Clearly, if $x_{1} \in T x_{1}$, we deduce that $x_{1}$ is a fixed point of $T$ and so we can conclude the proof. Now, we assume that $x_{1} \notin T x_{1}$ and hence $T x_{0} \neq T x_{1}$. Since $F \in \mathcal{F}_{s}$ is continuous from the right, there exists a real number $h>1$ such that

$$
F\left(h s H\left(T x_{0}, T x_{1}\right)\right)<F\left(s H\left(T x_{0}, T x_{1}\right)\right)+\tau .
$$

Next, from $d\left(x_{1}, T x_{1}\right)<h H\left(T x_{0}, T x_{1}\right)$, we deduce that there exists $x_{2} \in T x_{1}$ (obviously, $x_{2} \neq$ $\left.x_{1}\right)$ such that $d\left(x_{1}, x_{2}\right) \leq h H\left(T x_{0}, T x_{1}\right)$. Therefore, we can write

$$
F\left(\operatorname{sd}\left(x_{1}, x_{2}\right)\right) \leq F\left(\operatorname{shH}\left(T x_{0}, T x_{1}\right)\right)<F\left(s H\left(T x_{0}, T x_{1}\right)\right)+\tau,
$$

which implies

$$
\begin{aligned}
2 \tau+F\left(s d\left(x_{1}, x_{2}\right)\right) & \leq 2 \tau+F\left(s H\left(T x_{0}, T x_{1}\right)\right)+\tau \\
& \leq F\left(d\left(x_{0}, x_{1}\right)\right)+\tau .
\end{aligned}
$$

Consequently, we get

$$
\tau+F\left(s d\left(x_{1}, x_{2}\right)\right) \leq F\left(d\left(x_{0}, x_{1}\right)\right) .
$$

Iterating this procedure, we construct a sequence $\left\{x_{n}\right\} \subset X$ such that $x_{n} \notin T x_{n}, x_{n+1} \in T x_{n}$ and

$$
\tau+F\left(s d\left(x_{n+1}, x_{n+2}\right)\right) \leq F\left(d\left(x_{n}, x_{n+1}\right)\right) \text { for all } n \in \mathbb{N} \cup\{0\} .
$$


In order to simplify the reading of calculations, let $d_{n}=d\left(x_{n}, x_{n+1}\right)>0$ for all $n \in \mathbb{N} \cup\{0\}$. It follows by (3.2) and property (F4) that

$$
\tau+F\left(s^{n} d\left(x_{n}, x_{n+1}\right)\right) \leq F\left(s^{n-1} d\left(x_{n-1}, x_{n}\right)\right) \quad \text { for all } n \in \mathbb{N} \cup\{0\} .
$$

Thus, by (3.3), we have

$$
F\left(s^{n} d_{n}\right) \leq F\left(s^{n-1} d_{n-1}\right)-\tau \leq \cdots \leq F\left(d_{0}\right)-n \tau \quad \text { for all } n \in \mathbb{N},
$$

and so

$$
\lim _{n \rightarrow+\infty} F\left(s^{n} d_{n}\right)=-\infty
$$

which in view of property (F2) gives

$$
\lim _{n \rightarrow+\infty} s^{n} d_{n}=0
$$

Now, by property (F3) there exists $k \in(0,1)$ such that

$$
\lim _{n \rightarrow+\infty}\left(s^{n} d_{n}\right)^{k} F\left(s^{n} d_{n}\right)=0 .
$$

By (3.4), for all $n \in \mathbb{N}$, we get

$$
\begin{aligned}
\left(s^{n} d_{n}\right)^{k} F\left(s^{n} d_{n}\right)-\left(s^{n} d_{n}\right)^{k} F\left(d_{0}\right) & \leq\left(s^{n} d_{n}\right)^{k}\left(F\left(d_{0}\right)-n \tau\right)-\left(s^{n} d_{n}\right)^{k} F\left(d_{0}\right) \\
& =-n \tau\left(s^{n} d_{n}\right)^{k} \leq 0 .
\end{aligned}
$$

Passing to limit as $n \rightarrow+\infty$ in (3.5), we obtain

$$
\lim _{n \rightarrow+\infty} n\left(s^{n} d_{n}\right)^{k}=0
$$

and hence $\lim _{n \rightarrow+\infty} n^{1 / k} s^{n} d_{n}=0$. Now, the last limit implies that the series $\sum_{n=1}^{+\infty} s^{n} d_{n}$ is convergent and hence $\left\{x_{n}\right\}$ is a Cauchy sequence. Since $(X, d, s)$ is a complete $b$-metric space, then there exists $u \in X$ such that $\lim _{n \rightarrow+\infty} x_{n}=u$. Finally, we prove that $u$ is a fixed point of $T$, that is, $u \in T u$.

Firstly, we observe that if there exists an increasing sequence $\left\{n_{k}\right\} \subset \mathbb{N}$ such that $x_{n_{k}} \in T u$ for all $k \in \mathbb{N}$, since $T u$ is closed and $\lim _{k \rightarrow+\infty} x_{n_{k}}=u$, we deduce $u \in T u$ and hence the proof is completed. Then we assume that there exists $n_{0} \in \mathbb{N}$ such that $x_{n} \notin T u$ for all $n \in \mathbb{N}$ with $n \geq n_{0}$. It follows that $T x_{n-1} \neq T u$ for all $n \geq n_{0}$.

Now, using (3.1) with $x=x_{n}$ and $y=u$, we obtain

$$
2 \tau+F\left(s H\left(T x_{n}, T u\right)\right) \leq F\left(d\left(x_{n}, u\right)\right)
$$

which implies

$$
\begin{aligned}
2 \tau+F\left(d\left(x_{n+1}, T u\right)\right) & \leq 2 \tau+F\left(s H\left(T x_{n}, T u\right)\right) \\
& \leq F\left(d\left(x_{n}, u\right)\right) .
\end{aligned}
$$


Since $F$ is strictly increasing and $\tau \in \mathbb{R}^{+}$, we obtain

$$
d\left(x_{n+1}, T u\right)<d\left(x_{n}, u\right)
$$

Also, we have

$$
\begin{aligned}
d(u, T u) & \leq s\left[d\left(u, x_{n+1}\right)+d\left(x_{n+1}, T u\right)\right] \\
& \leq s d\left(u, x_{n+1}\right)+s d\left(x_{n}, u\right)
\end{aligned}
$$

and passing to limit as $n \rightarrow+\infty$ in the previous inequality, we get

$$
d(u, T u) \leq 0
$$

which implies $d(u, T u)=0$. Finally, since $T u$ is closed, we obtain that $u \in T u$, that is, $u$ is a fixed point of $T$.

As an application of Theorem 3.4 we get the following proof of Nadler's fixed point theorem in $b$-metric spaces [17].

Theorem 3.5 Let $(X, d, s)$ be a complete b-metric space and let $T: X \rightarrow C B(X)$. Assume that there exists $k \in(0,1)$ such that

$$
s H(T x, T y) \leq k d(x, y)
$$

for all $x, y \in X$. Then $T$ has a fixed point.

Proof Let $\tau \in \mathbb{R}^{+}$be such that $k=e^{-2 \tau}$. From (3.6), for all $x, y \in X$ with $T x \neq T y$, we get

$$
F(s H(T x, T y)) \leq-2 \tau+F(d(x, y))
$$

that is,

$$
2 \tau+F(s H(T x, T y)) \leq F(d(x, y))
$$

where $F(x)=\ln x$. Thus we can apply Theorem 3.4 to deduce that $T$ has a fixed point.

Remark 3.6 Let $C L(X)$ be the family of non-empty and closed subsets of $X$. Notice that Theorems 1.1 and 3.5 hold also in the case of a multivalued mapping $T: X \rightarrow C L(X)$.

\subsection{Feng-Liu type theorems}

Another very interesting approach to studying the existence of fixed points for multivalued mappings was proposed by Feng and Liu [16]. Here, we investigate the possibility to extend this approach to the $b$-metric setting. The main reason of this research is to obtain data dependence results for fixed points set. We recall some notions and fix notation as follows. 
Let $(X, d, s)$ be a $b$-metric space and let $T: X \rightarrow C L(X)$ be a multivalued mapping. Let Fix $(T):=\{x \in X: x \in T x\}$ denote the fixed point set of $T$. Also, define the function $f_{T}: X \rightarrow$ $\mathbb{R}$ as $f_{T} x=d(x, T x)$. Then, for a positive constant $\alpha \in(0,1)$ and each $x \in X$, define the set

$$
I_{\alpha}^{x}:=\{y \in T x: \alpha d(x, y) \leq d(x, T x)\} .
$$

Definition 3.7 Let $(X, d, s)$ be a $b$-metric space and let $T: X \rightarrow C L(X)$ be a multivalued mapping. A function $f: X \rightarrow \mathbb{R}$ is called $T$-lower semicontinuous if, for each $\left\{x_{n}\right\} \subset X$ with $x_{n+1} \in T x_{n}$ and $\lim _{n \rightarrow+\infty} x_{n}=x \in X$, we have

$$
f x \leq \liminf _{n \rightarrow+\infty} f x_{n} .
$$

Definition 3.8 Let $T: X \rightarrow C L(X)$ be a multivalued mapping. The graph of $T$ is the subset $\{(x, y): x \in X, y \in T x\}$ of $X \times X$; we denote the graph of $T$ by $G(T)$. Then $T$ is a closed multivalued mapping if the graph $G(T)$ is a closed subset of $\left(X \times X, d^{*}\right)$, where the metric $d^{*}$ is given by $d^{*}((x, y),(u, v))=d(x, u)+d(y, v)$ for all $(x, y),(u, v) \in X \times X$.

Now, we state and prove the following theorem.

Theorem 3.9 Let $(X, d, s)$ be a complete $b$-metric space and let $T: X \rightarrow C L(X)$ be a multivalued mapping. Suppose that there exists $r \in\left(0, s^{-1} \alpha\right)$ with $\alpha \in(0,1)$ such that for any $x \in X$ there is $y \in I_{\alpha}^{x}$ satisfying

$$
d(y, T y) \leq r d(x, y) .
$$

Then $T$ has a fixed point in $X$ provided that one of the following conditions holds:

(i) $f_{T}$ is $T$-lower semicontinuous,

(ii) $T$ is closed.

Proof Since $T x$ is a non-empty closed set for any $x \in X, I_{\alpha}^{x}$ is non-empty for any constant $\alpha \in(0,1)$. Now, for a fixed point $x_{0} \in X$, there exists $x_{1} \in I_{\alpha}^{x_{0}}$ such that

$$
d\left(x_{1}, T x_{1}\right) \leq r d\left(x_{0}, x_{1}\right) .
$$

If $x_{1}$ is not a fixed point of $T$, we choose $x_{2} \in I_{\alpha}^{x_{1}}$ such that

$$
d\left(x_{2}, T x_{2}\right) \leq r d\left(x_{1}, x_{2}\right)
$$

Again, if $x_{2}$ is not a fixed point of $T$ (and so on), by iterating this procedure, we can get an iterative sequence $\left\{x_{n}\right\}$, where $x_{n+1} \in I_{\alpha}^{x_{n}}$ and

$$
d\left(x_{n+1}, T x_{n+1}\right) \leq r d\left(x_{n}, x_{n+1}\right) \quad \text { for all } n \in \mathbb{N} \cup\{0\} .
$$

On the other hand, $x_{n+1} \in I_{\alpha}^{x_{n}}$ implies

$$
\alpha d\left(x_{n}, x_{n+1}\right) \leq d\left(x_{n}, T x_{n}\right) \quad \text { for all } n \in \mathbb{N} \cup\{0\} .
$$


The next step of the proof is to show that the sequence $\left\{x_{n}\right\}$ is a Cauchy sequence. Using (3.8) and (3.9), we get

$$
d\left(x_{n+1}, x_{n+2}\right) \leq \frac{r}{\alpha} d\left(x_{n}, x_{n+1}\right) \quad \text { for all } n \in \mathbb{N} \cup\{0\} .
$$

Since $r / \alpha<s^{-1}$, by Lemma 2.9 we deduce that $\left\{x_{n}\right\}$ is a Cauchy sequence and so, by completeness of the $b$-metric space $(X, d, s),\left\{x_{n}\right\}$ converges to some $u \in X$. Now we claim that $x$ is a fixed point of $T$. Therefore, we distinguish two cases.

Case 1: Suppose that (i) holds true. Again, by (3.8) and (3.9), we get

$$
d\left(x_{n+1}, T x_{n+1}\right) \leq \frac{r}{\alpha} d\left(x_{n}, T x_{n}\right) \quad \text { for all } n \in \mathbb{N} \cup\{0\},
$$

which implies

$$
d\left(x_{n}, T x_{n}\right) \leq\left(\frac{r}{\alpha}\right)^{n} d\left(x_{0}, T x_{0}\right) \quad \text { for all } n \in \mathbb{N} \cup\{0\} .
$$

Consequently,

$$
\liminf _{n \rightarrow+\infty} f_{T} x_{n}=\lim _{n \rightarrow+\infty} f_{T} x_{n}=\lim _{n \rightarrow+\infty} d\left(x_{n}, T x_{n}\right)=0 .
$$

Since $x_{n+1} \in T x_{n}, f_{T}$ is $T$-lower semicontinuous and $\lim _{n \rightarrow+\infty} x_{n}=u$, we have

$$
f_{T} u=d(u, T u)=0 .
$$

Since $T u$ is closed, we get that $u \in T u$, that is, $u$ is a fixed point of $T$.

Case 2: If (ii) holds true, then from $x_{n+1} \in T x_{n}$ for all $n \in \mathbb{N} \cup\{0\}$ and

$$
\lim _{n \rightarrow+\infty} d^{*}\left(\left(x_{n}, x_{n+1}\right),(u, u)\right)=\lim _{n \rightarrow+\infty}\left[d\left(x_{n}, u\right)+d\left(x_{n+1}, u\right)\right]=0,
$$

we get that $(u, u) \in \operatorname{Gr}(T)$ and hence $u \in T u$. Thus $u$ is a fixed point of $T$.

This completes the proof.

Now, we show that Theorem 3.9 is a generalization of the following version of Nadler's fixed point theorem in $b$-metric spaces.

Theorem 3.10 Let $(X, d, s)$ be a complete b-metric space and let $T: X \rightarrow C L(X)$ be a multivalued mapping such that for all $x, y \in X$, we have $H(T x, T y) \leq r d(x, y)$, where $r \in\left(0, s^{-1}\right)$, then $T$ has a fixed point.

Proof We have to show that all the hypotheses of Theorem 3.9 hold true. Firstly, we prove that $T$ satisfies condition (3.7) of Theorem 3.9. Indeed, for all $x \in X$ and $y \in T x$, we write

$$
d(y, T y) \leq H(T x, T y) \leq r d(x, y)
$$

and hence the assertion holds trivially for each $x \in X$ and $y \in I_{\alpha}^{x}$ with $\alpha \in(0,1)$ such that $r<\alpha s^{-1}$. It would remain to show that $f_{T}: X \rightarrow \mathbb{R}$ defined by $f_{T} x=d(x, T x)$ is $T$-lower 
semicontinuous. Indeed, let $\left\{x_{n}\right\} \subset X$ be a sequence with $x_{n+1} \in T x_{n}$ and $\lim _{n \rightarrow+\infty} x_{n}=$ $x \in X$. Clearly, we have

$$
d(x, T x) \leq s\left[d\left(x, x_{n+1}\right)+H\left(T x_{n}, T x\right)\right] \leq s\left[d\left(x, x_{n+1}\right)+r d\left(x_{n}, x\right)\right],
$$

and hence, passing to limit as $n \rightarrow+\infty$, we get $f_{T} x=0$. This implies that

$$
f_{T} x \leq \liminf _{n \rightarrow+\infty} f_{T} x_{n}
$$

This completes the proof.

Finally, we give a local version of Theorem 3.9.

Theorem 3.11 Let $(X, d, s)$ be a complete b-metric space, $x_{0} \in X, R>0$ and let $T: X \rightarrow$ $C L(X)$ be a multivalued mapping. Suppose that there exists $r \in\left(0, s^{-1} \alpha\right)$ with $\alpha \in(0,1)$ such that for any $x \in \bar{B}\left(x_{0}, R\right)$ there is $y \in I_{\alpha}^{x}$ satisfying

$$
d(y, T y) \leq r d(x, y) .
$$

If $d\left(x_{0}, T x_{0}\right) \leq \frac{\alpha}{s}\left(1-\frac{s r}{\alpha}\right) R$, then $T$ has a fixed point in $\bar{B}\left(x_{0}, R\right)$ provided one of the following conditions holds:

(i) $f_{T}$ is $T$-lower semicontinuous,

(ii) $T$ is closed.

Proof Proceeding as in the proof of Theorem 3.9, we construct an iterative sequence $\left\{x_{n}\right\}$ with initial point $x_{0}$, with $x_{n+1} \in I_{\alpha}^{x_{n}}$ and satisfying the conditions (3.8)-(3.10) for all $n \in$ $\mathbb{N} \cup\{0\}$. From (3.10) and $d\left(x_{0}, T x_{0}\right) \leq \frac{\alpha}{s}\left(1-\frac{s r}{\alpha}\right) R$, we obtain

$$
\begin{aligned}
d\left(x_{n}, x_{n+1}\right) & \leq\left(\frac{r}{\alpha}\right)^{n} d\left(x_{0}, x_{1}\right) \\
& \leq \frac{r^{n}}{\alpha^{n+1}} d\left(x_{0}, T x_{0}\right) \\
& \leq\left(\frac{r}{\alpha}\right)^{n} \frac{1}{s}\left(1-\frac{s r}{\alpha}\right) R
\end{aligned}
$$

for all $n \in \mathbb{N} \cup\{0\}$.

This implies $x_{n} \in \bar{B}\left(x_{0}, R\right)$. Indeed, we have

$$
\begin{aligned}
d\left(x_{0}, x_{n}\right) & \leq \sum_{k=1}^{n} s^{k} d\left(x_{k-1}, x_{k}\right) \\
& \leq \sum_{k=1}^{n} s^{k}\left(\frac{r}{\alpha}\right)^{k-1} \frac{1}{s}\left(1-\frac{s r}{\alpha}\right) R \\
& <\left(1-\frac{s r}{\alpha}\right) R \sum_{k=1}^{+\infty}\left(\frac{s r}{\alpha}\right)^{k-1}=R,
\end{aligned}
$$

and so $x_{n} \in \bar{B}\left(x_{0}, R\right)$. Thus, following the proof of Theorem 3.9, it is easy to show that $T$ has a fixed point in $\bar{B}\left(x_{0}, R\right)$. 


\section{Existence of solution for integral inclusions of Fredholm type}

In this section, we study the solvability of integral inclusions of Fredholm type. Precisely, we present an existence result of solution under general conditions on multivalued operators. For more on the solution of integral inclusions and related problems, the reader is referred to $[1,4,28]$ and the references therein.

Now, we consider the following integral inclusion of Fredholm type:

$$
x(t) \in f(t)+\int_{0}^{1} G(t, s, x(s)) d s \quad \text { for all } t \in[0,1]
$$

where $G:[0,1] \times[0,1] \times \mathbb{R} \rightarrow K_{c v}(\mathbb{R})$ is a multivalued operator, where $K_{c v}$ denotes the family of non-empty compact and convex subsets of $\mathbb{R}$.

Let $I=[0,1]$ and let $C(I, \mathbb{R})$ be the space of all continuous functions $f: I \rightarrow \mathbb{R}$. It is well known that such a space with the metric given by

$$
d(x, y)=\sup _{t \in I}(x(t)-y(t))^{2}=\left\|(x-y)^{2}\right\|_{\infty} \quad \text { for all } x, y \in C(I, \mathbb{R})
$$

is a complete $b$-metric space with $s=2$.

Adapting an idea in [4], we prove the following theorem.

\section{Theorem 4.1 Suppose that the following conditions hold:}

(i) for each $x \in C(I, \mathbb{R})$, the multivalued operator $G: I \times I \times \mathbb{R} \rightarrow K_{c v}(\mathbb{R})$ is such that $G(t, s, x(s))$ is lower semicontinuous in $I \times I$;

(ii) $f \in C(I, \mathbb{R})$;

(iii) there exists $l(t, \cdot) \in L^{1}(I)$, for each $t \in I$ and $\sup _{t \in I} \int_{0}^{1} l(t, s) d s \leq \sqrt{\frac{k}{2}}$ with $k \in(0,1)$, such that

$$
H(G(t, s, x), G(t, s, y)) \leq l(t, s)|x(s)-y(s)|
$$

for all $t, s \in I$ and for all $x, y \in \mathbb{R}$.

Then the integral inclusion (4.1) has at least one solution in $C(I, \mathbb{R})$.

Proof Let us define the multivalued operator $T: C(I, \mathbb{R}) \rightarrow C L(C(I, \mathbb{R}))$ by

$$
T x(t)=\left\{v \in C(I, \mathbb{R}) \text { such that } v(t) \in f(t)+\int_{0}^{1} G(t, s, x(s)) d s, t \in I\right\}
$$

for each $x \in C(I, \mathbb{R})$. Let $x \in C(I, \mathbb{R})$ and denote $G_{x}(t, s):=G(t, s, x(s)), t, s \in I$. For the multivalued operator $G_{x}: I \times I \rightarrow K_{c v}(\mathbb{R})$, by Michael's selection theorem, we get that there exists a continuous operator $g_{x}: I \times I \rightarrow \mathbb{R}$ such that $g_{x}(t, s) \in G_{x}(t, s)$ for all $t, s \in I$. This implies that $f(t)+\int_{0}^{1} g_{x}(t, s) d s \in T x$ and so $T x$ is a non-empty set. It is an easy matter to show that $T x$ is closed, and so details are omitted (see also [4]). This implies that $T x$ is closed in $(C(I, \mathbb{R}), d)$.

Next, we show that the multivalued operator $T$ satisfies all the hypotheses of Theorem 3.5. To this aim, let $x, y \in C(I, \mathbb{R})$ be such that $v \in T x$. Then there exists $g_{x}(t, s) \in G_{x}(t, s)$ with $t, s \in I$ such that $v(t)=f(t)+\int_{0}^{1} g_{x}(t, s) d s, t \in I$. On the other hand, by hypothesis (iii), 
we get

$$
H(G(t, s, x(s)), G(t, s, y(s))) \leq l(t, s)|x(s)-y(s)|
$$

for all $t, s \in I$ and for all $x, y \in \mathbb{R}$. Consequently, there exists $w(t, s) \in G_{y}(t, s)$ such that

$$
\left|g_{x}(t, s)-w(t, s)\right| \leq l(t, s)|x(s)-y(s)|
$$

for all $t, s \in I$. Now, we can consider the multivalued operator $S$ defined by

$$
S(t, s)=G_{y}(t, s) \cap\left\{u \in \mathbb{R} \text { such that }\left|g_{x}(t, s)-u\right| \leq l(t, s)|x(s)-y(s)|\right\}
$$

for all $t, s \in I$. Taking into account the fact that the multivalued operator $G$ is lower semicontinuous, it follows that there exists a continuous operator $g_{y}: I \times I \rightarrow \mathbb{R}$ such that $g_{y}(t, s) \in S(t, s)$ for all $t, s \in I$. We have

$$
z(t)=f(t)+\int_{0}^{1} g_{y}(t, s) d s \in f(t)+\int_{0}^{1} G(t, s, y(s)) d s, \quad t \in I
$$

and

$$
\begin{aligned}
|v(t)-z(t)|^{2} & \leq\left(\int_{0}^{1}\left|g_{x}(t, s)-g_{y}(t, s)\right| d s\right)^{2} \\
& \leq\left(\int_{0}^{1} l(t, s)|x(s)-y(s)| d s\right)^{2} \\
& \leq\left(\int_{0}^{1} l(t, s) \sqrt{(x(s)-y(s))^{2}} d s\right)^{2} \\
& \leq\left(\int_{0}^{1} l(t, s) \sqrt{\left\|(x-y)^{2}\right\|_{\infty}} d s\right)^{2} \\
& \leq\left\|(x-y)^{2}\right\|_{\infty}\left(\int_{0}^{1} l(t, s) d s\right)^{2}
\end{aligned}
$$

for all $t \in I$. Thus, $d(v, z) \leq \frac{k}{2} d(x, y)$. Interchanging the roles of $x$ and $y$, we obtain that

$$
2 H(T x, T y) \leq k d(x, y)
$$

for all $x, y \in C(I, \mathbb{R})$. Thus, all the conditions of Theorem 3.5 are satisfied and hence we deduce the existence of a solution of (4.1).

Remark 4.2 Consider the following differential inclusion:

$$
x^{\prime}(t) \in G(t, s, x(s)), \quad t, s \in I,
$$

where $x \in C(I, \mathbb{R})$ and $G: I \times I \times \mathbb{R} \rightarrow K_{c v}(\mathbb{R})$ is a multivalued operator. Notice that (4.2) is equivalent to (4.1) with $f(t)=0$. Consequently, if the operator $G$ satisfies the hypotheses of Theorem 4.1, then the existence of a solution of (4.2) follows by an application of Theorem 4.1. 
Example 4.3 For all $x \in C(I, \mathbb{R})$ and $t, s \in I$, let $G(t, s, x(s))=\left\{v \in \mathbb{R}: g_{1}(t, s, x(s)) \leq v \leq\right.$ $\left.g_{2}(t, s, x(s))\right\}$, where $g_{1}(t, s, x(s))$ is upper semicontinuous in $I \times I$ and $g_{2}(t, s, x(s))$ is lower semicontinuous in $I \times I$. Consider the following differential inclusion:

$$
x^{\prime}(t) \in G(t, s, x(s)), \quad t, s \in I
$$

and assume that there exists $l(t, \cdot) \in L^{1}(I)$, for each $t \in I$ and $\sup _{t \in I} \int_{0}^{1} l(t, s) d s \leq \frac{\sqrt{2}}{2}$, such that

$$
\max \left|g_{1}(t, s, x(s))-g_{2}(t, s, y(s))\right| \leq l(t, s)|x(s)-y(s)|
$$

for all $t, s \in I$ and for all $x, y \in \mathbb{R}$.

Clearly, the multivalued operator $G$ is compact and convex valued. Thus, all the hypotheses of Theorem 4.1 are satisfied with $f(t)=0$, and hence the above two-point boundary value problem has at least one solution.

\section{Stability of solutions set for fixed point problems}

We study data dependence of solutions set for fixed point problems by using the technique presented in Section 3.2. Indeed, in view of Theorem 3.9, we prove a data dependence theorem of the fixed points set for two multivalued mappings.

Theorem 5.1 Let $(X, d, s)$ be a complete b-metric space and let $S, T: X \rightarrow C L(X)$ be two multivalued mappings such that $\sup _{x \in X} H(S x, T x)<+\infty$. Suppose that there exists $r \in\left(0, s^{-1} \alpha\right)$ with $\alpha \in(0,1)$ such that for any $x \in X$ there is $y \in I_{\alpha}^{x}$ satisfying

$$
d(y, S y) \leq r d(x, y) \quad \text { and } \quad d(y, T y) \leq r d(x, y) .
$$

Then we have

$$
H(\operatorname{Fix}(S), \operatorname{Fix}(T)) \leq \frac{s}{\alpha-r s} \sup _{x \in X} H(S x, T x)
$$

provided that one of the following conditions holds:

(i) $f_{S}$ and $f_{T}$ are, respectively, $S$-lower and T-lower semicontinuous,

(ii) $S$ and $T$ are closed.

Proof By Theorem 3.9 we deduce that $\operatorname{Fix}(S)$ and $\operatorname{Fix}(T)$ are non-empty sets. Also, notice that $\operatorname{Fix}(S)$ and $\operatorname{Fix}(T)$ are closed. Indeed, for instance, let $\left\{u_{n}\right\} \subset \operatorname{Fix}(S)$ be a sequence such that $\lim _{n \rightarrow+\infty} u_{n}=u$. Then, if $S$ is closed, the conclusion follows easily. On the other hand, if $f_{S} x:=d(x, S x)$ is $S$-lower semicontinuous, then we have

$$
0 \leq d(u, S u)=f_{S} u \leq \liminf _{n \rightarrow+\infty} f_{S} u_{n}=\liminf _{n \rightarrow+\infty} d\left(u_{n}, S u_{n}\right)=0 .
$$

It follows that $u \in S u$, that is, $u \in \operatorname{Fix}(S)$. The same holds for $\operatorname{Fix}(T)$.

Let us consider $x_{0} \in \operatorname{Fix}(S)$. Hence, there exists $x_{1} \in I_{\alpha}^{x_{0}}$ with $d\left(x_{1}, T x_{1}\right) \leq r d\left(x_{0}, x_{1}\right)$. Now, since $\alpha d\left(x_{0}, x_{1}\right) \leq d\left(x_{0}, T x_{0}\right)$, we obtain

$$
d\left(x_{0}, x_{1}\right) \leq \alpha^{-1} d\left(x_{0}, T x_{0}\right) \quad \text { and } \quad d\left(x_{1}, T x_{1}\right) \leq r d\left(x_{0}, x_{1}\right) \leq r \alpha^{-1} d\left(x_{0}, T x_{0}\right) .
$$


Iterating this process, we can construct an iterative sequence $\left\{x_{n}\right\}$ such that

(i) $x_{0} \in \operatorname{Fix}(S)$,

(ii) $d\left(x_{n}, T x_{n}\right) \leq\left(r \alpha^{-1}\right)^{n} d\left(x_{0}, T x_{0}\right)$,

(iii) $d\left(x_{n}, x_{n+1}\right) \leq \alpha^{-1}\left(r \alpha^{-1}\right)^{n} d\left(x_{0}, T x_{0}\right)$.

From (iii), we deduce that $\left\{x_{n}\right\}$ is a Cauchy sequence and therefore it converges to an element $u \in X$. From (ii), by following the same lines of proof as in Theorem 3.9, we get that $u \in \operatorname{Fix}(T)$. Again, if $m>n$ from

$$
d\left(x_{n}, x_{m}\right) \leq \frac{s\left(r \alpha^{-1}\right)^{n}}{\alpha-r s} d\left(x_{0}, T x_{0}\right),
$$

letting $m \rightarrow+\infty$, we deduce

$$
d\left(x_{n}, u\right) \leq \frac{s\left(r \alpha^{-1}\right)^{n}}{\alpha-r s} d\left(x_{0}, T x_{0}\right)
$$

for each $n \in \mathbb{N} \cup\{0\}$. Then, for $n=0$, we get

$$
\begin{aligned}
d\left(x_{0}, u\right) & \leq \frac{s}{\alpha-r s} d\left(x_{0}, T x_{0}\right) \\
& \leq \frac{s}{\alpha-r s} H\left(S x_{0}, T x_{0}\right) \\
& \leq \frac{s}{\alpha-r s} \sup _{x \in X} H(S x, T x) .
\end{aligned}
$$

In a similar way we can prove that, for each $y_{0} \in \operatorname{Fix}(T)$, there exists $v \in \operatorname{Fix}(S)$ such that

$$
d\left(y_{0}, v\right) \leq \frac{s}{\alpha-r s} \sup _{x \in X} H(S x, T x) .
$$

Thus, the proof is complete.

Building on Theorem 5.1 and dealing with a sequence of multivalued mappings, we obtain the following result.

Theorem 5.2 Let $(X, d, s)$ be a complete b-metric space and let $T_{i}: X \rightarrow C L(X)$ be a sequence of multivalued mappings. Suppose that there exists $r \in\left(0, s^{-1} \alpha\right)$ with $\alpha \in(0,1)$ such that for any $x \in X$ there is $y \in I_{\alpha}^{x}$ satisfying $d\left(y, T_{i} y\right) \leq r d(x, y)$ and $\lim _{i \rightarrow+\infty} H\left(T_{i} x, T_{0} x\right)=0$ uniformly, where $i \in \mathbb{N} \cup\{0\}$. Then we have

$$
\lim _{i \rightarrow+\infty} H\left(\operatorname{Fix}\left(T_{i}\right), \operatorname{Fix}\left(T_{0}\right)\right)=0 .
$$

Proof Let $\varepsilon>0$ be arbitrary and choose $N \in \mathbb{N}$ such that

$$
\sup _{x \in X} H\left(T_{i} x, T x\right)<\frac{\alpha-r s}{s} \varepsilon
$$

for $i \geq N$ and for each $x \in X$. Consequently, by Theorem 5.1 we get

$$
H\left(\operatorname{Fix}\left(T_{i}\right), \operatorname{Fix}\left(T_{0}\right)\right)<\varepsilon
$$

for $i \geq N$. Therefore, for the arbitrarity of $\varepsilon$, the proof is complete. 


\section{Competing interests}

The authors declare that they have no competing interests.

\section{Authors' contributions}

All authors contributed equally and significantly in writing this article. All authors read and approved the final manuscript.

\section{Author details}

${ }^{1}$ Dipartimento di Matematica e Informatica, Università degli Studi di Palermo, Via Archirafi, 34, Palermo, 90123, Italy.

${ }^{2}$ Department of Mathematics, College of Science, King Saud University, P.O. Box 2455, Riyadh, 11451, Saudi Arabia.

\section{Authors' information}

Calogero Vetro is a member of the Gruppo Nazionale per I'Analisi Matematica, la Probabilità e le loro Applicazioni (GNAMPA) of the Istituto Nazionale di Alta Matematica (INdAM).

\section{Acknowledgements}

The authors would like to extend their sincere appreciation to the Deanship of Scientific Research at King Saud University for its funding of this research through the International Research Group Project No. IRG14-04.

Received: 3 January 2015 Accepted: 22 April 2015 Published online: 14 May 2015

\section{References}

1. Cabada, A, Infante, G, Tojo, FAF: Nontrivial solutions of Hammerstein integral equations with reflections. Bound. Value Probl. 2013, Article ID 86 (2013)

2. Agarwal, RP, O'Regan, D, Wong, PJY: Existence results of Brezis-Browder type for systems of Fredholm integral inclusions. Adv. Differ. Equ. 2011, Article ID 43 (2011)

3. Petruşel, A: Integral inclusions. Fixed point approaches. Comment. Math. Prace Mat. 40, 147-158 (2000)

4. Sîntămărian, A: Integral inclusions of Fredholm type relative to multivalued $\varphi$-contractions. In: Seminar on Fixed Point Theory Cluj-Napoca, vol. 3, pp. 361-368 (2002)

5. Nadler, SB: Multivalued contraction mappings. Pac. J. Math. 30, 475-488 (1969)

6. Abbas, M, Ali, B, Vetro, C: A Suzuki type fixed point theorem for a generalized multivalued mapping on partial Hausdorff metric spaces. Topol. Appl. 160, 553-563 (2013)

7. Amini-Harandi, A: Fixed point theory for set-valued quasi-contraction maps in metric spaces. Appl. Math. Lett. 24, 1791-1794 (2011)

8. Altun, I, Minak, G, Dağ, H: Multi-valued F-contractions on complete metric spaces. J. Nonlinear Convex Anal. 16 659-666 (2015)

9. Aydi, H, Abbas, M, Vetro, C: Partial Hausdorff metric and Nadler's fixed point theorem on partial metric spaces. Topol. Appl. 159, 3234-3242 (2012)

10. Chifu, C, Petruşel, G: Existence and data dependence of fixed points and strict fixed points for contractive-type multivalued operators. Fixed Point Theory Appl. 2007, Article ID 34248 (2007)

11. Daffer, PZ, Kaneko, H: Fixed points of generalized contractive multi-valued mappings. J. Math. Anal. Appl. 192 655-666 (1995)

12. Reich, S: Approximate selections, best approximations, fixed points, and invariant sets. J. Math. Anal. Appl. 62, 104-113 (1978)

13. Reich, S: Fixed points of contractive function. Boll. Unione Mat. Ital. 5, $26-42$ (1972)

14. Wardowski, D: Fixed points of a new type of contractive mappings in complete metric spaces. Fixed Point Theory Appl. 2012, Article ID 94 (2012)

15. Sgroi, M, Vetro, C: Multi-valued F-contractions and the solution of certain functional and integral equations. Filomat 27, 1259-1268 (2013)

16. Feng, Y, Liu, S: Fixed point theorems for multi-valued contractive mappings and multi-valued Caristi type mappings. J. Math. Anal. Appl. 317, 103-112 (2006)

17. Czerwik, S: Nonlinear set-valued contraction mappings in b-metric spaces. Atti Semin. Mat. Fis. Univ. Modena 46 263-276 (1998)

18. Bakhtin, IA: The contraction mapping principle in quasimetric spaces. In: Functional Analysis, vol. 30, pp. 26-37. Ul'yanovsk. Gos. Ped. Inst., Ul'yanovsk (1989) (in Russian)

19. Berinde, V: Generalized contractions in quasimetric spaces. In: Seminar on Fixed Point Theory, pp. 3-9 (1993)

20. Boriceanu, M, Bota, M, Petruşel, A: Multivalued fractals in b-metric spaces. Cent. Eur. J. Math. 8, 367-377 (2010)

21. Boriceanu, M, Petruşel, A, Rus, IA: Fixed point theorems for some multivalued generalized contractions in $b$-metric spaces. Int. J. Math. Stat. 6, 65-76 (2010)

22. Bota, M, Molinár, A, Varga, C: On Ekeland's variational principle in b-metric spaces. Fixed Point Theory 12, 21-28 (2011)

23. Cosentino, M, Salimi, P, Vetro, P: Fixed point results on metric-type spaces. Acta Math. Sci. 34, 1237-1253 (2014)

24. Czerwik, S, Dlutek, K, Singh, SL: Round-off stability of iteration procedures for set-valued operators in $b$-metric spaces. J. Natur. Phys. Sci. 11, 87-94 (2007)

25. Paesano, D, Vetro, P: Fixed point theorems for $\alpha$-set-valued quasi-contractions in $b$-metric spaces. J. Nonlinear Convex Anal. 16, 685-696 (2015)

26. Aydi, H, Bota, M-F, Karapinar, E, Mitrović, S: A fixed point theorem for set-valued quasicontractions in b-metric spaces. Fixed Point Theory Appl. 2012, Article ID 88 (2012)

27. Czerwik, S: Contraction mappings in b-metric spaces. Acta Math. Univ. Ostrav. 1, 5-11 (1993)

28. Infante, G, Pietramala, P: Perturbed Hammerstein integral inclusions with solutions that change sign. Comment. Math. Univ. Carol. 50, 591-605 (2009) 